\title{
Esophageal cancer in the Sudan: focus on the screening for ebv and the surgical management
}

\begin{abstract}
Objective: The aim of this study was to screen for the existence of Epstein Barr Virus (EBV) in esophageal cancer (EC) tissue samples obtained from Sudanese patients and to review the available literature associated with surgical management.
\end{abstract}

Methodology: In this retrospective study 102 formalin fixed paraffin wax embedded tissue's blocks were retrieved from histopathology laboratories in Khartoum City, Sudan and subsequently tested by Immunohistochemical methods for the presence of EBV. All specimens were previously diagnosed as having esophageal carcinoma.

Results: As regards Immuno histochemistry (IHC) staining for EBV, positive findings were revealed in $22(21.5 \%)$ and $79 /(78.5 \%)$ of the study subjects were negative. Of the 55 males with esophageal cancer, 15/55 (27\%) were identified with EBV infection and the remaining 40/55(73\%) found without EBV infection. Of the 46 females with esophageal cancer, $7 / 46(13 \%)$ were found positive for EBV immuno staining and the remaining 39/46(87\%) were found negative for EBV.

Conclusion: EBV might have some etiological effects contributing to the occurrence of esophageal cancer in Sudan. Crucial preventive strategies are extremely needed to reduce the burden of esophageal cancer in Sudan.

Keywords: esophageal cancer, Sudan, EBV, etiological effects, esophageal cancer
Volume 4 Issue 6 - 2017

\author{
Hussain Gadelkarim Ahmed, 1,2 Mohmad \\ Ahmad Babikir Ibraheem Beely, ${ }^{3}$ Mohammed \\ Siddig Abd El Aziz, ${ }^{4}$ Bassam Ahmed \\ ALmutlaq,' Majid Zannon Alturkstani ${ }^{5}$ \\ 'Department of Pathology, University of Hail, Saudi Arabia \\ 2Department of Histopathology and Cytology, University of \\ Khartoum, Sudan \\ ${ }^{3}$ Department of Medical Laboratory, University of Kordofan, \\ Sudan \\ ${ }^{4}$ Department of Histopathology and Cytology, Sudan University \\ for Science and Technology, Sudan
}

${ }^{5}$ Batterjee Medical College, Saudi Arabia

Correspondence: Professor Hussain Gadelkarim Ahmed, Department of Pathology, College of Medicine, 2440, University of Hail, Kingdom of Saudi Arabia (KSA),

Email hussaingad1972@yahoo.com

Received: May 07, 2017 | Published: September 07, 2017
Abbreviations: EC, esophageal cancer; IHC, immuno histochemistry; EBV, epstein barr virus; CMV. cytomegalovirus; HSV, herpes simplex virus

\section{Introduction}

Esophageal cancer (EC) occurs commonly, particularly in Asia, and it is the sixth leading cause of cancer deaths worldwide. Esophageal cancer classically happens in one of two forms, squamous cell carcinomas (SCCs) arising from the stratified squamous epithelial lining of the organ, and adenocarcinoma (AC) arising from columnar glandular cells that replace the squamous epithelium. ${ }^{2}$ Sarcomas and small cell carcinomas usually constitute less than $1 \%-2 \%$ of all esophageal cancers. ${ }^{3}$ The major risk factors for esophageal cancer include; gastro-esophageal reflux, ${ }^{4}$ tobacco smoking, alcohol drinking, ${ }^{5}$ viruses, ${ }^{6}$ susceptibility genes, ${ }^{7}$ low levels of intake of fruits and vegetables. ${ }^{8}$ The relationship between viral infection and esophageal cancer was previously reported for several viruses including; high human Papillomavirus (HR-HPV) particularly subtype 16, herpes simplex virus 1 (HSV-1), Epstein-Barr virus (EBV) and cytomegalovirus (CMV). However, the mechanisms of the viral action in addition to geographic variation in infection rates of these viruses still need more efforts. ${ }^{9} \mathrm{EBV}$ is an oncogenic virus that has been described to be linked to Burkitt's lymphoma, Hodgkin's disease, breast cancer, gastric carcinoma, nasopharyngeal carcinoma and leiomyosarcoma. ${ }^{10}$ However, whether EBV is associated to esophageal SCC still controversial: there are limited studies and their conclusions vary. ${ }^{11}$

\section{Surgical management}

Although esophageal cancer is the most common gastrointestinal (GI) cancer in the Sudan, but there a deep absence of literature from the Sudan, particularly in regard to surgical management. In a recent study a total of 114 patients were enrolled in the study, with a mean age of 47 years SD +/- 19 and a male to female ratio of 1: 1.04 . Malignant causes were mainly due to esophageal cancer (40.4\%) and cancer of the stomach cardia (3.5\%).Therapeutic intervention was attempted in $83 \%$ of the cases. ${ }^{12} \mathrm{~A}$ recent published study investigated 100 consecutive patients who experienced esophagectomy in Shaab Hospital in Khartoum during the period June 2003-Aug 2007. The mean age was $55 \pm 14$ years with an equal sex ratio. Fifty five per cent of patients presented with stage III\&IV locally advanced and or metastatic disease. Sixty seven percent of the patients underwent a 2 -stage resection, Lewis Tanner type while $27 \%$ underwent a 3-stage resection, McKeon operation and $6 \%$ had total gastrectomy with distal esophagectomy and roux-en-y reconstruction. The 30days postoperative mortality was $10 \%$. In 75 patients who could be traced, the overall 5years survival was $21 \%(\mathrm{n}=16)$ and the 10 years survival was $8 \%(n=6) .{ }^{13}$ Therefore, the aim of this study was to screen for the presence of EBV in esophageal cancer tissue samples obtained from Sudanese patients and to review the available literature associated with surgical management.

\section{Materials and methods}

In this study we investigated 102 Sudanese patients with esophageal carcinomas, 55 were males and 46 were females (males'/females' ratio, 1.20:1.00), their ages ranged between 12 and 98years with mean 
age of 60years old. These patients with esophageal carcinomas, were examined for the existence of HR-HPV subtypes 16\&18 using LMP1 (Latent membrane protein 1 (LMP1) of EBV) Antibody immuno histochemistry. The diagnosis of esophageal cancer was established depending on clinical examination and histopathological features of the biopsy. EC diagnosis was confirmed base on Royal College of Pathologists criteria (Royal College of Pathologists, 2005). The esophageal cancer including 91/102(89.2\%) squamous cell carcinomas (SCCs) and 11/102(10.8\%) adenocarcinoma. The sample included full coverage of patients with esophageal lesions referred to Radiation and isotope Centre Khartoum with available sample quantity and data. Ethical consent was approved from ethical committee of the Faculty Research Board and Hospital. LMP1 immuno histochemistry (IHC) was performed on formalin-fixed paraffin embedded (FFPE) tissue sections using kits from (Dako Agilent Technologies Company). Following antigen retrieval using Citrate buffer $(\mathrm{pH} 6)$ for 30minutes, sections were incubated with mouse monoclonal LMP1, and then Envision System HRP anti-mouse was employed, followed by diaminobenzidine (DAB) chromogenthen counterstained with Harris' Hematoxylin. Known LMP1-positive sections were used as positive controls, and sections without addition of primary antibody were used as negative controls. All LMP1 IHC tissue sections were semi quantitatively evaluated by two examiners for concentration of staining in the cytoplasm and nucleus of the cells. Intensity of the staining was counted as 0 (none), + (weak), ++ (moderate), or +++ (strong), with 0 or + scores regarded as negative and ++ or +++ considered as positive. LMP1 scoring was done without knowledge of EBV infection status.

\section{Results}

The present study retrieved 102 tissue blocks prepared from esophageal carcinoma's specimens (91patients were with squamous cell carcinoma and 11 were with adenocarcinoma). Of the 102 study subjects 55 were males and 46 were females their age ranging from 12 to 98 years old with a mean age of 60 years. The majority of the study populations were at the age range 66-75 years constituting 26 patients followed by age ranges, both $56-65$ and $<45$ years representing 24 patients. Moreover, 16 patients were identified among age group 46-55years, hence only 12patients were found among age group $76+$ years, as indicated in Figure 1.

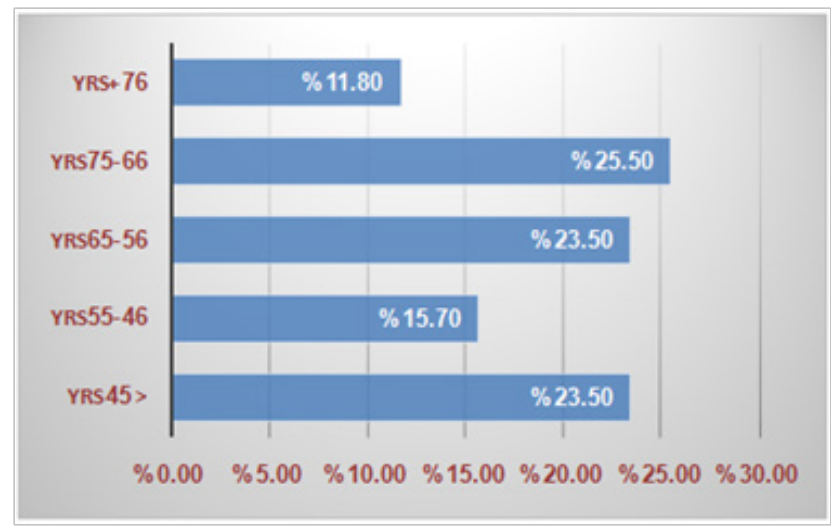

Figure I Description of the study population by age.

Regarding IHC staining for EBV, positive findings were revealed in $22(21.5 \%)$ and $79 /(78.5 \%)$ of the study subjects were negative, as shown in Microphotographs $1 \& 2$. Of the 55 males with esophageal cancer, 15/55 (27\%) were identified with EBV infection and the remaining $40 / 55(73 \%)$ found without EBV infection. Of the 46 females with esophageal cancer, $7 / 46(13 \%)$ were found positive for EBV immunostaining and the remaining 39/46(87 \%) were found negative for EBV. The association of EBV risk with male sex was $\mathrm{OR}(\mathrm{CI})=2.08(0.77-5.7), \mathrm{P}=0.145$. According to cancer type, of the 91 cases of the Squamous cell carcinoma, 19/91(21\%) were found with positive EBV and the remaining $72 / 91(79 \%)$ were negative. Of the 11 cases of the Adenocarcinoma, 3(27.3\%) were found with positive EBV and the remaining 8(72.7\%) were negative, as indicated in Table 1. The association of EBV risk with Squamous cell carcinoma was $\mathrm{OR}(\mathrm{CI})=0.70(0.17-2.9), \mathrm{P}=0.63$, as indicated in Table $1 \&$ Figure 2.

Table I Distribution of IHC results of EBV by sex and cancer type

\begin{tabular}{llll}
\hline Sex & Positive & Negative & Total \\
\hline Males & 15 & 40 & 55 \\
Females & 7 & 39 & 46 \\
Total & 22 & 79 & 101
\end{tabular}

Cancer Type

Squamous 19 72 91 Adenocarcinoma 3 8

Total 22

80

102

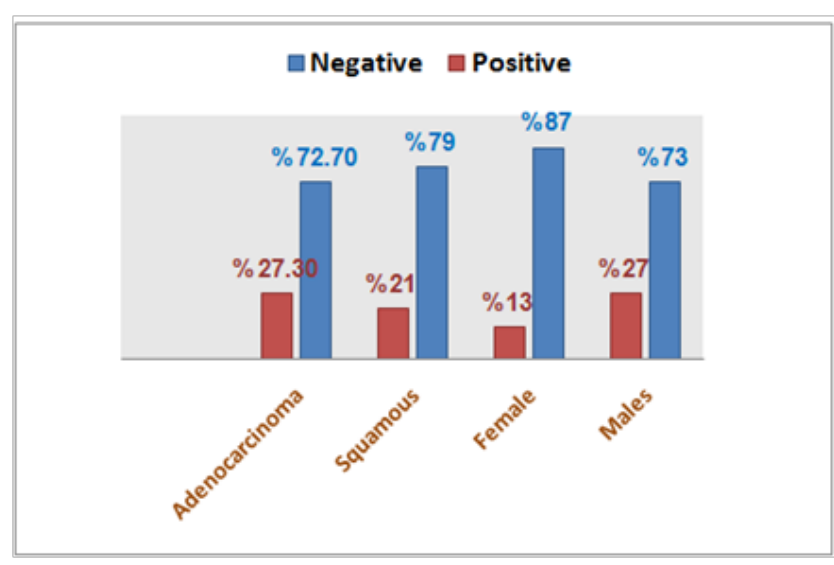

Figure 2 Description of IHC results of EBV by sex and cancer type.

Concerning IHC staining results of EBV with age distribution, the highest positive results were found in age group 56-65years, representing $7 / 23(32 \%)$, followed by $46-55,<45,66-75$ and $76+$ constituting $6 / 22(27.3 \%), 4 / 22(18.2 \%), 3 / 22(13.5 \%)$ and $2 / 22(9 \%)$ respectively, whereas; the groups with the highest negative results were 66-75 years, representing 23/80(28.8\%) followed by $<45,56-$ 65 and (46-55\& 76+) constituting 20/80 (25\%), 17/80(21.2\%), and $10 / 80(12.5 \%)$ for each, respectively, as shown in Table 2 \& Figure 3. 
Table 2 Distribution of $\mathrm{IHC}$ results of EBV by age

\begin{tabular}{llll}
\hline Age group & Positive & Negative & Total \\
\hline$<45$ years & 4 & 20 & 24 \\
$46-55$ & 6 & 10 & 16 \\
$56-65$ & 7 & 17 & 24 \\
$66-75$ & 3 & 23 & 26 \\
$76+$ & 2 & 10 & 12 \\
Total & 22 & 80 & 102 \\
\hline
\end{tabular}



76+yrs 66-75yrs56-65yrs46-55yrs <45yrs

Figure 3 Description of $\mathrm{IHC}$ results of EBV by age.

\section{Discussion}

The prevalence rates of esophageal cancer vary according to geographic regions and related risk factors. Many risk factors, including EBV, may be involved in the development of esophageal cancer. Although there is no a clear-cut association between EBV infection and EC, but there is evidence that EBV infection may contribute to esophageal cancer in certain high-risk populations. ${ }^{12}$ However, in the present study we found a prevalence of $21.5 \%$ of infection of EBV among Sudanese patients with EC. Relatively higher prevalence rates were previously reported in some studies. In study from German investigated 37 patients with esophageal carcinoma (Esophageal squamous cell carcinomas $(n=23)$ and adenocarcinoma $(n=14)$ ) for the presence of human EBV DNA, EBV was detected in $35 \%$ of squamous cell carcinomas and $36 \%$ of adenocarcinoma. ${ }^{12}$ Nevertheless in the present study EBV positive was found in $27.3 \%$ of the cases of the adenocarcinoma and $21 \%$ of the cases of SCC. In another study an association between EBV infection and the development of esophageal carcinoma has been reported in $35.5 \%$ of the cases. ${ }^{13}$ Although, It is strongly suspected that the EBV plays a role in the genesis of nasopharyngeal carcinoma, but some studies reported that, EBV is infrequently associated with esophageal SCC, and may appear through tumor-infiltrating lymphocytes in some advanced lesions..$^{14,15}$ However, the Prevalence rates of EBV in gastroesophageal cancer vary between 5 and $20 \%$, which may depend on the patient groups in different studies. ${ }^{16-18}$ EBV-associated tumorigenesis appears to be rather restricted to gastric cancer while the role of EBV in other parts such as esophageal carcinomas appears to be insignificant in most parts of the globe. ${ }^{19}$ To the best of our knowledge there no study investigated the role of the EBV in the etiology of EC from Sudan. Most of the studies in this context from Sudan, tested the association between EBV and nasopharyngeal carcinoma. Almost all of these studies found some degrees of association between EBV and nasopharyngeal carcinoma. In one study from Sudan, EBV genes were detected in $92 / 150(61.3 \%)$ of patients with nasopharyngeal carcinoma. ${ }^{20}$ Another study from Sudan has tested 43 Sudanese patients with nasopharyngeal carcinoma for the presence of EBV using EBER-ISH. All nasopharyngeal carcinoma biopsies (100\%) were positive for EBER1 in almost all carcinoma cells. ${ }^{21}$ These studies may give some degree of suspicion for $\mathrm{EBV}$ as possible etiological factor contributing to the etiology of EC in Sudan. In regard to the sex, the prevalence of EBV infection in the current study was $27 \%$ in males and $13 \%$ in females. Since there are only small studies in regard to the relationship between EBV and EC, the relationship of sex and EBV can't be strongly, but studies on the association of EBV and nasopharyngeal carcinoma indicates similar findings to our series, though the association between the number of males and females was not statistically significant. In regard to the age most of cases with positive EBV expression were found among age 56-65years. Although cancer in general accumulate at elderly people, but this need further research in regard to the start of infection and initiation of carcinogenesis. ${ }^{22,23}$

Esophagectomy is the leading treatment for esophageal cancer. Esophagectomy can be achieved by several diverse procedures, including McKeown (cervico-thoraco-abdominal), Ivor-Lewis (thoraco-abdominal), and transhiatal methodologies. It has been proposed that minimally invasive esophagectomy contributes to the decline of pulmonary complications. Cervico-thoraco-abdominal 3 -field lymphadenectomy may extend survival, but a randomized control study on this matter has not been conducted. Death and disease rates after esophagectomy persist high. A number of meta-analyses verified that esophagectomy at low-volume hospitals was related with a significant increase in the incidence of in-hospital and 30-day mortality. The influence of hospital volume on long-standing outcome remains to be an issue of debate..$^{24}$ However, in the Sudan, there was great improvement in the early postoperative mortality from $27 \%$ in 1986 to $10 \%$ in recent reports. The surgical treatment presented a quick symptoms of relief which suited most patients coming from distant locations and couldn't afford to stay for extended periods in the Capital. ${ }^{12}$ The limitations in the present study include; its retrospective settings and that fact it didn't measure other factors that contribute to the etiology of EC cancer, such as smoking, alcohol use, and other viruses (HPV). The lack of surgical management data was extra limitation in the present study. In conclusion: EBV might have some etiological effects contributing to the occurrence of esophageal cancer in Sudan. Crucial preventive strategies are extremely needed to reduce the burden of esophageal cancer in Sudan. Esophageal cancer is highly aggressive; consequently, a multimodality approach is essential to cure this disease. Surgical resection remains the backbone among these approaches.

\section{Acknowledgements}

None. 


\section{Conflict of interest}

The author declares no conflict of interest.

\section{References}

1. Yuwei Zhang. Epidemiology of esophageal cancer. World J Gastroentero. 2013;19(34):5598-5606.

2. Blot WJ, Devesa SS, Fraumeni JF. Continuing climb in rates of esophageal adenocarcinoma: an update. JAMA. 1993;270(11):1320.

3. Kwatra KS, Prabhakar BR, Jain S, et al. Sarcomatoid carcinoma (carcinosarcoma) of the esophagus with extensive areas of osseous differentiation: a case report. Indian J Pathol Microbiol. 2003;46(1):4951.

4. Pohl H, Wrobel K, Bojarski C, et al. Risk factors in the development of esophageal adenocarcinoma. Am J Gastroenterol. 2013;108(2):200-207.

5. Ye XH, Huai JP, Ding J, et al. Smoking, alcohol consumption, and the risk of extrahepatic cholangiocarcinoma: a meta-analysis. World $J$ Gastroenterol. 2013;19(46):8780-878.

6. Xi R, Pan S, Chen X, et al. HPV16 E6-E7 induces cancer stem-like cells phenotypes in esophageal squamous cell carcinoma through the activation of PI3K/Akt signaling pathway in vitro and in vivo. Oncotarget. 2016;7(35):57050-57065.

7. Palles C, Findlay JM, Tomlinson I. Common variants confer susceptibility to barrett's esophagus: insights from the first genome-wide association studies. Adv Exp Med Biol. 2016;908:265-290.

8. Wang A, Zhu C, Fu L, et al. Citrus fruit intake substantially reduces the risk of esophageal cancer: a meta-analysis of epidemiologic studies. Medicine (Baltimore). 2015;94(39):e1390.

9. Zhang DH, Zhang QY, HongCQ, et al. Prevalence and association of human papillomavirus 16, Epstein-Barr virus, herpes simplex virus-1 and cytomegalovirus infection with human esophageal carcinoma: a casecontrol study. Oncol Re. 2011;25(6):1731-1738.

10. Thompson MP, Kurzrock R. Epstein-Barr virus and cancer. Clin Cancer Res. 2004;10(3):803-821.

11. Wenjia XU, Zhongshun LIU, Qunchao BAO, et al. Viruses, Other pathogenic microorganisms and esophageal cancer. Gastrointestinal Tumors. 2015;2(1):2-13.
12. Mudawi HM, Mahmoud AO, El Tahir MA, et al. Use of endoscopy in diagnosis and management of patients with dysphagia in an African setting. Dis Esophagus. 2010;23(3):196-200.

13. Ahmed ME, Mahadi SI, Ali BM. The surgical treatment of esophageal cancer in Sudan: A 100 consecutive cases. Int J Surg. 2016;29:101-107.

14. Awerkiew S, Bollschweiler E, Metzger R, et al. Esophageal cancer in Germany is associated with Epstein-Barr-virus but not with papillomaviruses. Med Microbiol Immunol. 2003;192(3):137-140.

15. Wang LS, Chow KC, Wu YC, et al. Detection of Epstein-Barr virus in esophageal squamous cell carcinoma in Taiwan. Am J Gastroenterol. 1999;94(10):2834-2839.

16. Yanai H, Hirano A, Matsusaki K, et al. Epstein-Barr virus association is rare in esophageal squamous cell carcinoma. Int $J$ Gastrointest Cancer. 2003;33(2-3):165-170

17. Chen PC, Pan CC, Hsu WH, et al. Epstein-Barr virus-associated lymphoepithelioma-like carcinoma of the esophagus. Hum Pathol. 2003;34(4):407-411.

18. Lee JH, Kim SH, Han $\mathrm{SH}$, et al. Clinicopathological and molecular characteristics of Epstein-Barr virus-associated gastric carcinoma: a metaanalysis. J Gastroenterol Hepatol. 2009;24(3):354-365.

19. Murphy G, Pfeiffer R, Camargo MC, et al. Meta-analysis shows that prevalence of 19-Epstein-Barr virus-positive gastric cancer differs based on sex and anatomic location. Gastroenterology. 2009;137(3):824-833.

20. Nishikawa J, Yoshiyama H, Iizasa H, et al. Epstein-barr virus in gastric carcinoma. Cancers (Basel). 2014;6(4):2259-2274.

21. Sarbia M, ZurHausen A, Feith M. Esophageal (Barrett's) adenocarcinoma is not associated with Epstein-Barr virus infection: an analysis of 162 cases. Int J Cancer. 205;117(4):698-700.

22. Ahmed HG, Suliman RS, Abd El Aziz MS, et al. Molecular screening for Epstein Barr virus (EBV) among Sudanese patients with nasopharyngeal carcinoma (NPC). Infectious Agents and Cancer. 2015;10:6.

23. Adam AAM, Abdullah NE, El Hassan LAM, et al. Detection of Epstein - Barr virus in Nasopharyngeal carcinomain Sudanese by in situ hybridization. J Cancer Ther. 2014;5:517-522.

24. Watanabe M, Baba Y, Yoshida N, et al. Surgical treatment for esophageal cancer. J Nucl Med Radiat Ther. 2002;S2:004. 